\title{
Safety factor in single human motor end-plates studied in vivo with single fibre electromyography
}

\author{
ERIK STÅLBERG, HANS H. SCHILLER, AND MARTIN S. SCHWARTZ \\ From the Department of Clinical Neurophysiology, University Hospital, Uppsala, Sweden
}

\begin{abstract}
SYNOPSIS In a single fibre EMG investigation on healthy subjects the relationship between the neuromuscular jitter and the safety factor of the motor end-plate relative to curare was studied. A positive correlation was found between the jitter increase after a standard dose of D-tubocurarine and the initial jitter. The conclusion was drawn that the neuromuscular jitter can be used as an indicator of the safety factor. There are a number of nerve-muscle disorders in which the jitter is increased, probably indicating a reduced safety factor which may be of clinical importance when these patients are subjected to diseases or drugs which would otherwise not produce neuromuscular symptomatology.
\end{abstract}

The safety factor for neuromuscular transmission is normally high (Waser, 1960; Paton and Waud, 1967) and therefore minor disturbances cannot be demonstrated in vivo. When there is a reduced safety factor, as in myasthenia gravis and in other disorders with motor endplate involvement, certain drugs without effect on the normal motor end-plate may worsen the neuromuscular transmission.

With the method of single fibre electromyography (SFEMG) (Ekstedt, 1964; Stålberg, 1966; Stålberg and Ekstedt, 1973), it is possible to study the neuromuscular transmission in situ, by measuring the variability of the neuromuscular transmission time in individual motor endplates, expressed as the jitter (Stålberg et al., 1971). With various pharmacological agents (Ekstedt and Stålberg, 1969; Stålberg et al., 1973) and in neuromuscular diseases (Stålberg and Ekstedt, 1973) the jitter is increased to abnormal values before impulse blockings occur, when neuromuscular disturbances in clinical and conventional neurophysiological tests become evident. The jitter increases with increasing impairment of the transmission.

The aim of the present investigation was to study the relationship between the neuromus-

(Accepted 15 April 1975.) cular jitter and the safety factor of the motor end-plate.

\section{METHODS}

SINGLE FIBRE ELECTROMYOGRAPHY (SFEMG) Recordings SFEMG recordings were made with a SFEMG electrode with platinum recording surface $25 \mu \mathrm{m}$ in diameter. The needle was inserted into the slightly voluntarily activated right extensor digitorum communis III muscle (EDC) and a position was sought where action potentials from two fibres belonging to the same motor unit, a potential pair, could be obtained for determination of the neuromuscular jitter (Stålberg et al., 1971; Ekstedt et al., 1974). The subjects were asked to keep a relatively stable innervation rate between 8 and $15 \mathrm{~Hz}$ aided by a ratemeter and a loudspeaker.

Jitter The jitter, expressed as the mean consecutive differences (MCD) of interpotential intervals (Ekstedt et al., 1974) was calculated with a PDP 11-40 computer either on-line or off-line for groups of $\mathbf{5 0}$ discharges. All recordings were stored on analogue tape (AKAI X330).

The interpotential intervals were measured with an electronic counter (HP 5326B). The most stable point was chosen on each of the action potentials for the measurements, usually on the steep fast positivenegative deflection as close to the zero intersection as possible with regard to noise and interfering action potentials. Erroneous data were omitted by setting 
upper and lower limits for acceptable values. The results from the computer were displayed on a typewriter, storage oscilloscope and $x-y$ plotter.

Also the mean value of the interpotential intervals (MIPI) for each potential pair was calculated and displayed for 25,50 , or 100 discharges. The innervation rate was measured for the corresponding number of discharges.

COMMENTS ON JITTER VALUES The measured jitter is the vector sum of the two Gaussian distributed and independent time variations in two motor end-plates belonging to the same motor unit. The jitter of the individual motor end-plates can be determined by single muscle fibre recordings after nerve stimulation but these values cannot be obtained with voluntary

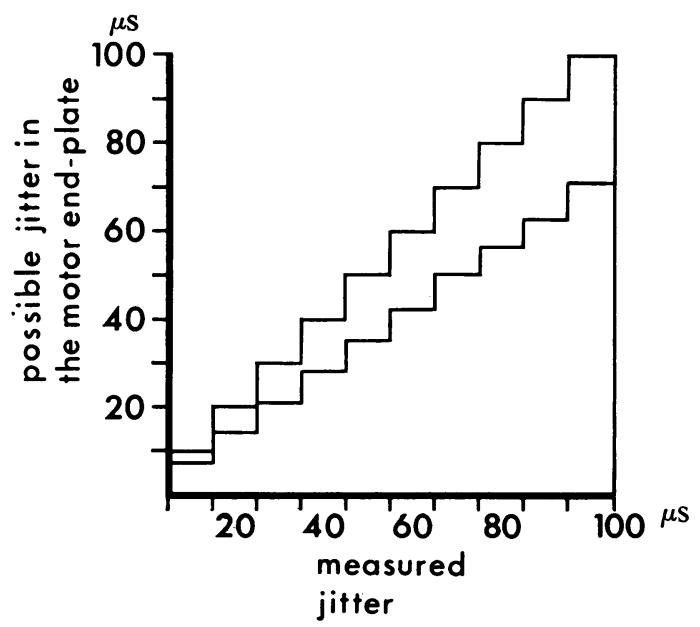

FIG. 1 Schematic representation of the calculated possible jitter values for the individual motor end-plates comprising a measured action potential pair, according to the formula $\mathrm{x}=\sqrt{{\mathrm{y_{1 }}}^{2}+\mathrm{y}_{2}^{2}} \cdot \mathrm{x}=$ measured jitter value, y calculated jitter for an individual motor endplate. Area between steplines = jitter range for the endplate with the higher jitter; lower part = jitter range of the end-plate with the lower jitter.

activity. In this situation, a range of possible combination of values can be calculated

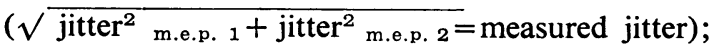
m.e.p. $=$ motor end-plate

(see Fig. 1). For example, in a recording with a measured jitter value of $30 \mu \mathrm{s}$, the motor end-plate with the lower jitter may have a value from $2 \mu s$ (the minimal jitter recorded in one motor end-plate after nerve stimulation) to maximally $22 \mu \mathrm{s}$ when the other has a jitter ranging from 29 to $22 \mu \mathrm{s}$. Thus the motor end-plate with the higher jitter at this level is never less than $22 \mu \mathrm{s}$.

EXPERIMENTS Either $0.5 \mathrm{mg}$ or $30 \mu \mathrm{g} / \mathrm{kg}$ D-tubocurarine in $10 \mathrm{ml}$ saline was injected intravenously into the left arm during the continuous SFEMG recording from the right EDC which had begun three to five minutes previously.

DECREMENT MEASUREMENTS Surface recordings were made from the right abductor digiti quinti muscle at electrical supramaximal stimulation at $2 \mathrm{~Hz}$ and pulse width $0.1 \mathrm{~ms}$ of the ulnar nerve at the wrist. In the other arm, $0.5 \mathrm{mg}$ or $30 \mu \mathrm{g} / \mathrm{kg}$ D-tubocurarine was injected intravenously. The decrement was measured as the percentage drop in amplitude of the negative peak of the muscle action potential between the first and fourth response.

SUBJECTS Investigations were performed on 11 healthy subjects aged $25-45$ years. The time between two investigations on the same subject always ex=o ceeded 24 hours.

\section{RESULTS}

SFEMG WITH LOW DOSE OF D-TUBOCURARINE Im five recordings $0.5 \mathrm{mg}$ D-tubocurarine wa\$ slowly injected intravenously into the left armo $\overrightarrow{0}$ Minimal clinical symptoms were reported, prin cipally short-lasting difficulties in focusing vision but no diplopia. There was no weakness of either hand.

The initial jitter values ranged from 14 to $30 \mu \mathrm{s}$ and there was no change in the jitter during the three to four minutes preceding the injection of $0.5 \mathrm{mg}$ D-tubocurarine. Thirty seconds after injection the jitter increased by 2-7 $\mu$ s with the maximal value at three to six minutes and the effect was not observed 10 minutes later. Each potential was maintained for about 15 minutes. There was a slight tendency for a greater increase to occur in those potential pairs with initially high jitter values. The change in mean interpotential interval (MIPI) during the experiment was less than $100 \mu \mathrm{s}$. In two experiments the innervation rate became irregular.

SFEMG WITH HIGH DOSE OF D-TUBOCURARINE In 11 recordings $30 \mu \mathrm{g} / \mathrm{kg}$ D-tubocurarine was slowly injected intravenously. All subjects re- 


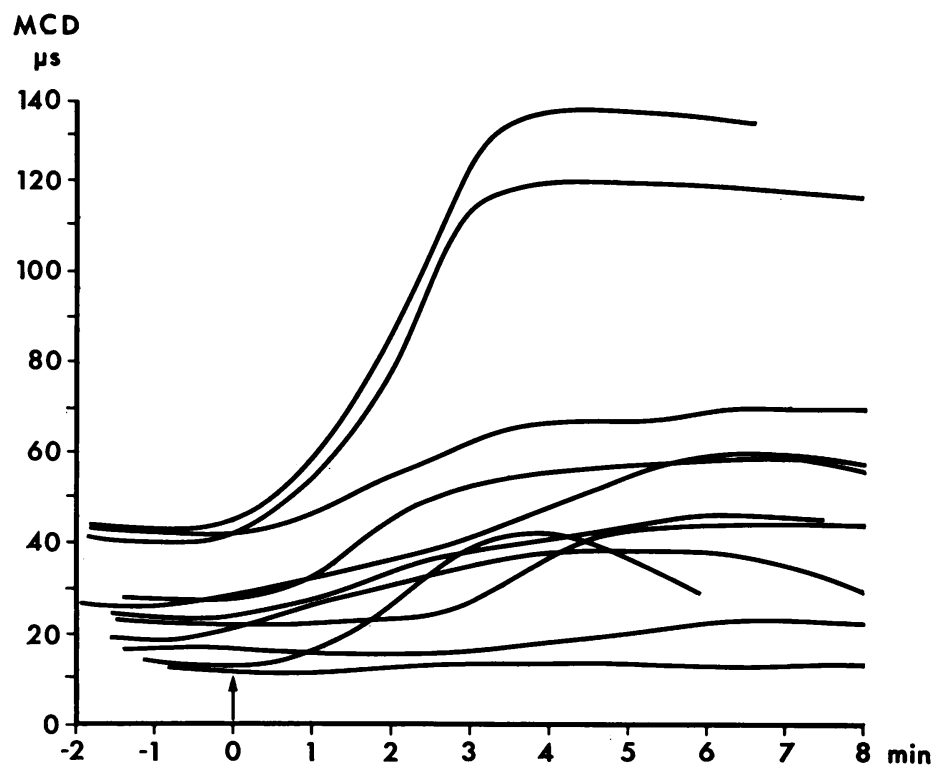

FIG. 2 The effect of $30 \mu \mathrm{g} / \mathrm{kg}$ intravenous $D$-tubocurarine on potential pairs in a normal EDC muscle. Arrow. indicates time of injection. The effect is more pronounced in potential pairs with higher initial jitter (MCD).

$\triangle M C D$

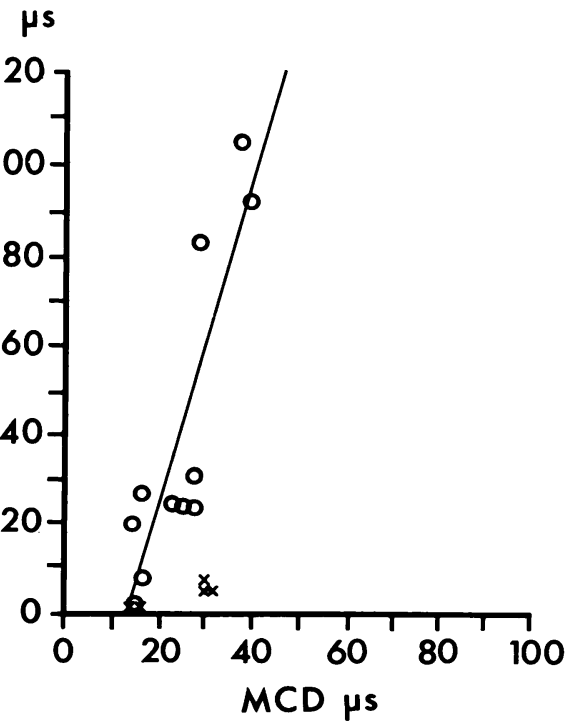

FIG. 3 Effect of $0.5 \mathrm{mg}(\times)$ and $30 \mu \mathrm{g} / \mathrm{kg}(\mathrm{C})$ of $\mathrm{D}$ tubocurarine on motor end-plates in the EDC muscle. The correlation coefficient for $\bigcirc$ is 0.83 . ported slight clinical symptoms with heavy eyelids and double vision predominating, usually lasting for three to four minutes. No weakness of the finger extensors was observed. The pretreatment jitter values ranged from 12 to $43 \mu \mathrm{s}$. The jitter was maximal three to five minutes after injection and ranged from 15 to $138 \mu \mathrm{s}$. The greatest increase was obtained in potential pairs with the highest initial jitter (Fig. 2). The increased jitter values usually persisted for several minutes. In three recordings nearly maximal values were found even after 10 minutes. Blockings were observed when the jitter exceeded values of about $80 \mu \mathrm{s}$. The MIPI changed maximally $500 \mu \mathrm{s}$. The innervation rate usually became irregular. A summary of the effects of the two doses is represented in Fig. 3. The correlation coefficient for the linear regression for the higher dose is 0.83 .

Surface recordings The decremental response was measured in three investigations with $0.5 \mathrm{mg}$ and in two with $30 \mu \mathrm{g} / \mathrm{kg}$ D-tubocurarine. There was no effect with either dose.

SIMULATION A series of electronic simulations were made to depict an intracellular recording in the end-plate zone to demonstrate some of the 

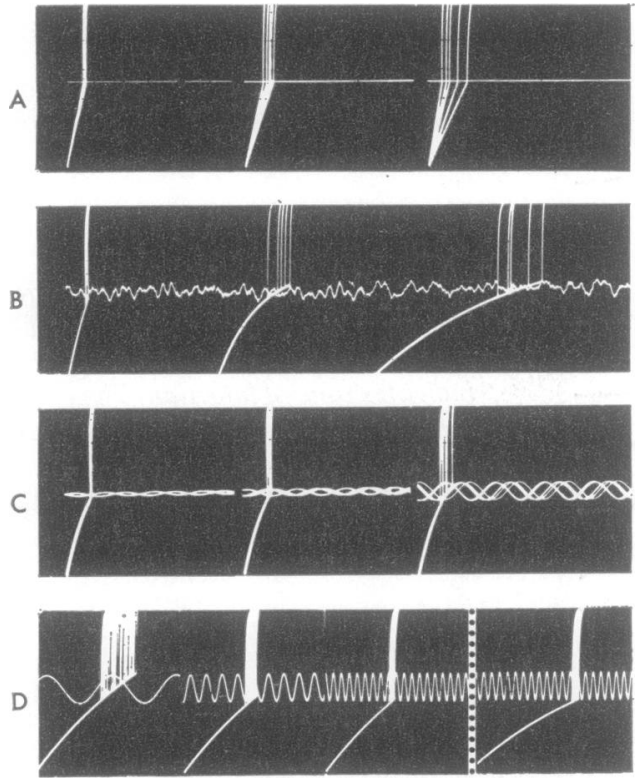

FIG. 4 Simulated intracellular recordings showing EPP and threshold level. A: Influence of a consecutive change in rise time of EPP with constant trigger level. There is an increased jitter with increased rise time variability. B: Effect of threshold fluctuation with constant EPP rise time. Three different rise times are represented. There is an increased jitter with increased rise time of the EPP. C: Effect of increasing amplitude of the threshold fluctuation with constant EPP rise time. Jitter increases with increasing amplitude. D: Effect of changes in frequency content of the threshold fluctuation. The jitter is smaller when the frequency of the fluctuation is higher. Last part shows that there is minimal effect on the jitter with slowed rise time with high frequency fluctuations.

factors possibly responsible for the neuromuscular jitter. The shape of the end-plate potential (EPP) was altered as well as the characteristics of the threshold (trigger level) for the firing of a muscle action potential. In particular, we studied the slope of the end-plate potential and types of threshold fluctuations (frequency content and amplitude).

End-plate potential With a constant trigger level a jitter was present when the slope of the EPP was varied for consecutive discharges (Fig. 4A). For an EPP with a rise time to the trigger level of $500 \mu \mathrm{s}$ a variation of $10 \%$ caused a variability range of $50 \mu \mathrm{s}$.

A small noise on the trigger level produced a jitter for consecutive impulses even with a constant shape of the end-plate potential (Fig. 4B). With a stepwise reduction of the slope of the EPP, usually corresponding to a decrease in amplitude (Thesleff and Quastel, 1965), the jitter initially showed only a minimal increase which progressively became more pronounced for each unit change of the EPP.

Trigger level fluctuations With a constant and relatively low frequency of the fluctuations the jitter increased with their increasing amplitude. A variability of $25 \mu \mathrm{s}$ was obtained if the amplitude of the noise was $3 \mathrm{mV}$ (the trigger level was set at $-60 \mathrm{mV}$ and the rise time of the EPP to the trigger level at $150 \mu \mathrm{s}$; the figures chosen to represent typical data obtained from intracellular recordings).

With a constant shape of the EPP, the jitter was changed by varying the frequency content of $v$ the threshold fluctuations. When the frequencs exceeded a certain value the jitter diminished (Fig. 4D). There were only slight changes of the jitter relative to the slope of the EPP (Fig. 4D) when the threshold fluctuations were of higlo frequency.

\section{DISCUSSION}

The aim of the present investigation was to study whether the range of jitter values for motor endplates in a normal muscle indicates differences in their safety factor relative to D-tubocurarine. Experiments of neuromuscular transmission in vitro (Del Castillo and Katz, 1954; Elmqvist and Quastel, 1965; Hubbard and Wilson, 1973) have shown that there are several hundred quanta of acetylcholine released with each nerve impulse, many times greater than that necessary to elicit a depolarization of the muscle fibre membrane. The safety factor expressed in this way is calculated to be four times in motor end-plates in rat diaphragm (Waser, 1960) and four to 12 times in the cat (Paton and Waud, 1967).

As shown in the presented simulation experiments, the jitter values can theoretically be due $\stackrel{N}{\sigma}$ to many different factors, some of which are not $N$ correlated with the safety factor. If these latter 
were most important for determining the jitter value, there should not be a greater effect of $D$ tubocurarine on motor end-plates with high jitter than on those with low jitter.

D-tubocurarine is known to block cholinergic receptors (Waser, 1960) and recent data (Hubbard and Wilson, 1973) indicate in addition a presynaptic effect seen as a reduction in the quantum content due to interference with acetylcholine release. The end-plate potential is reduced in amplitude and increased in rise time with D-tubocurarine (Thesleff and Quastel, 1965). In the present study, it was shown that the effect on the jitter was greater with the higher doses of D-tubocurarine, but still no decrement could be detected with surface recordings. More striking was the finding that there was a positive correlation between the initial jitter and its increase with D-tubocurarine. It should be noted that the measurements were always made from two motor end-plates, which introduces some dispersion of data presented in Fig. 3 as the effect on the two motor end-plates probably differs.

The obtained correlation between jitter and Dtubocurarine effect may indicate that the principal factors determining the jitter value are correlated with the safety factor. The motor endplates with high jitter probably have EPPs with a slow rise time and low amplitude.

The mean interpotential interval (MIPI) between the two action potentials in the potential pairs represents the difference in the propagation time for the two impulses along the nerve twigs from the last common branching point, at the neuromuscular junctions and along the muscle fibre to the recording site. The change in MIPI after D-tubocurarine, up to $500 \mu \mathrm{s}$ is mainly due to the different effect on the EPPs in the two motor end-plates, as this drug does not affect the impulse propagation velocity of the muscle fibre (Ekstedt and Stålberg, 1969) and there is no direct evidence that it affects the nerve fibres.

The tendency for an irregular innervation frequency with D-tubocurarine might be due to the subject's distraction during the drug effect. It could also indicate an effect on the muscle spindles (unpublished observation) resulting in a reduced afferent activity from the muscle. We have observed similar difficulties with innervation in patients with myasthenia gravis.

The different jitter for individual motor end- plates found in the normal muscle-probably indicating differences in EPP rise time-may be in accordance with the findings of Nastuk (1971), who found a fourfold variation in the rise time of the EPP in frog between different motor endplates.

The difference in the EPPs, which may be responsible for the range in jitter values, might be due to physiological differences such as amount of acetylcholine released with each nerve impulse, numbers of cholinergic receptors, and properties of the post-synaptic membrane. The difference in EPPs may also be due to the variation in structural characteristics such as nerve terminal size, postsynaptic area of folds and clefts and number of synaptic vesicles (Santa and Engel, 1973).

Since the jitter value seems to indicate the safety factor of the neuromuscular transmission, it can be questioned whether in the normal muscle the motor end-plates with high jitter are more susceptible to toxic (insecticides), metabolic (uraemia), and immunological (myasthenia) influences? Related to this question is the following observation. It has been demonstrated that in a number of disorders the jitter is increased because of a more or less pronounced disturbance in the neuromuscular transmissionfor example, in myasthenia gravis (Stålberg et al., 1974), in myasthenic syndrome (Schwartz and Stålberg, 1975) and with reinnervation such as polyneuropathies (Thiele and Stålberg, 1972), and in lower motor neurone disorders (Stålberg et al., 1975). Increased jitter in these conditions may indicate a reduced safety factor which may be of clinical importance when these patients are subjected to diseases or drugs which would otherwise not produce neuromuscular symptomatology.

The investigation was supported by the Swedish Medical Research Council (Grant No. 135). H.H.S. was supported by a grant from the Schweizerische Stiftung für Med. Biol. Stipendien. Kerstin Mellqvist lent technical assistance.

\section{REFERENCES}

DelCastillo, J., and Katz, B. (1954). Quantal components of the end plate potential. Journal of Physiology, 124, 560-573.

Ekstedt, J. (1964). Human single muscle fibre action potentials. Acta Physiologica Scandinavica, 61, suppl. 226.

Ekstedt, J., Nilsson, G., and Stålberg, E. (1974). Calculation of the electromyographic jitter. Journal of Neurology, Neurosurgery, and Psychiatry, 37, 526-539. 
Ekstedt, J., and Stålberg, E. (1969). The effect of non-paralytic doses of d-Tubocurarine of individual motor endplates in man, studied with a new electrophysiological method. Electroencephalography and Clinical Neurophysiology, 27, 557-562.

Elmqvist, D., and Quastel, D. M. J. (1965). A quantitative study of end-plate potentials in isolated human muscle. Journal of Physiology, 178, 5-29.

Hubbard, J. I., and Wilson, D. F. (1973). Neuromuscular transmission on a mammalian preparation on the absence of blocking drugs and the effect of D-tubocurarine. Journal of Physiology, 228, 307-325.

Nastuk, W. L. (1971). Mechanisms of neuromuscular blockade. Annals of New York Academy of Sciences, 183, 171182, 200.

Paton, W. M. D., and Waud, D. R. (1967). The margin of safety of neuromuscular transmission. Journal of Physiology, 191, 59-90.

Santa, T., and Engel, A. G. (1973). Histometric analysis of neuromuscular junction ultrastructure in rat red, white and intermediate muscle fibres. In New Developments in Electromyography and Clinical Neurophysiology, vol. 1, pp. 41-54. Edited by J. E. Desmedt. Karger: Basel.

Schwartz, M. S., and Stålberg, E. (1975). Myasthenic syndrome studied with single fibre electromyography. Archives of Neurology. (In press.)

Stålberg, E. (1966). Propagation velocity in human muscle fibres in situ. Acta Physiologica Scandinavica, 70, suppl. 287.

Stålberg, E., and Ekstedt, J. (1973), Single fibre EMG and microphysiology of the motor unit in normal and diseased muscle. In: New Developments in Electromyography and Clinical Neurophysiology, vol. 1, pp. 113-129. Edited by J. E. Desmedt. Karger: Basel.

Stålberg, E., Ekstedt, J., and Broman, A. (1971). The electromyographic jitter in normal human muscles. Electroencephalography and Clinical Neurophysiology, 31, 429-438.

Stålberg, E., Ekstedt, J., and Broman, A. (1974). Neuromuscular transmission in myasthenia gravis studied with single fibre electromyography. Journal of Neurology, Neurosurgery, and Psychiatry, 37, 540-547.

Stålberg, E., Schwartz, M. S., and Trontelj, J. V. (1975). Single fibre electromyography in various processes affecting the anterior horn cell. Journal of Neurological Sciences. 24, 403-415.

Stålberg, E., Thiele, B., and Hilton-Brown, P. (1973). Effect of succinylcholine on single motor end-plates in man. Acta Anaesthesiologica Scandinavica, 17, 108-118.

Thiele, B., and Stålberg, E. (1972). The bimodal jitter: a single fibre electromyographic finding. Journal of Neurology, Neurosurgery, and Psychiatry, 37, 403-411.

Thesleff, S., and Quastel, D. M. J. (1965). Neuromuscular pharmacology. Annual Review of Pharmacology, 5, 263284.

Waser, P. (1960). The cholinergic receptor. Journal of Pharmacy and Pharmacology, 12, 577-594. 